\title{
Adipose-Derived Mesenchymal Stem Cells do not Affect the Invasion and Migration Potential of Oral Squamous Carcinoma Cells
}

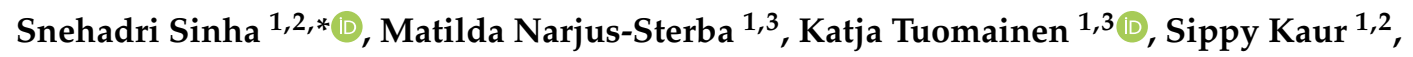 \\ Riitta Seppänen-Kaijansinkko ${ }^{1,2}$, Tuula Salo ${ }^{1,2,3,4,5}$, Bettina Mannerström ${ }^{1,2}$ and \\ Ahmed Al-Samadi ${ }^{1,3}$ (D) \\ 1 Department of Oral and Maxillofacial Diseases, Clinicum, University of Helsinki, 00014 Helsinki, Finland; \\ matilda.narjus-sterba@helsinki.fi (M.N.-S.); katja.tuomainen@helsinki.fi (K.T.); sippy.kaur@helsinki.fi (S.K.); \\ riitta.seppanen-kaijansinkko@helsinki.fi (R.S.-K.); tuula.salo@helsinki.fi (T.S.); \\ bettina.mannerstrom@helsinki.fi (B.M.); ahmed.al-samadi@helsinki.fi (A.A.-S) \\ 2 Helsinki University Central Hospital, 00290 Helsinki, Finland \\ 3 Translational Immunology Research Program (TRIMM), Faculty of Medicine, University of Helsinki, \\ 00014 Helsinki, Finland \\ 4 Cancer and Translational Medicine Research Unit, University of Oulu, 90014 Oulu, Finland \\ 5 Medical Research Centre, Oulu University Hospital, 90014 Oulu, Finland \\ * Correspondence: snehadri.sinha@helsinki.fi
}

Received: 28 July 2020; Accepted: 2 September 2020; Published: 4 September 2020

\begin{abstract}
Mesenchymal stem cells (MSCs) are commonly isolated from bone marrow and adipose tissue. Depending on the tissue of origin, MSCs have different characteristics and physiological effects. In various cancer studies, MSCs have been found to have either tumor-promoting or tumor-inhibiting action. This study investigated the effect of adipose tissue-MSCs (AT-MSCs) and bone marrow-MSCs (BM-MSCs) on global long interspersed nuclear element-1 (LINE-1) methylation, the expression level of microenvironment remodeling genes and cell proliferation, migration and invasion of oral tongue squamous cell carcinoma (OTSCC). Additionally, we studied the effect of human tongue squamous carcinoma (HSC-3)-conditioned media on LINE-1 methylation and the expression of microenvironment remodeling genes in AT-MSCs and BM-MSCs. Conditioned media from HSC-3 or MSCs did not affect LINE-1 methylation level in either cancer cells or MSCs, respectively. In HSC-3 cells, no effect of MSCs-conditioned media was detected on the expression of ICAM1, ITGA3 or $M M P 1$. On the other hand, HSC-3-conditioned media upregulated ICAM1 and MMP1 expression in both types of MSCs. Co-cultures of AT-MSCs with HSC-3 did not induce proliferation, migration or invasion of the cancer cells. In conclusion, AT-MSCs, unlike BM-MSCs, seem not to participate in oral cancer progression.
\end{abstract}

Keywords: mesenchymal stem cells; adipose tissue; bone marrow; oral squamous cell carcinoma; invasion; migration 


\section{Introduction}

Mesenchymal stem cells (MSCs) comprise a heterogeneous source of adherent cells that express specific surface markers, e.g., CD73, CD90 and CD105, and have the capacity for multilineage differentiation [1]. MSCs release various cytokines and growth factors that, through paracrine signaling, modulate a range of acute and chronic pathological conditions [2,3]. Though MSCs are present in all organs of the body, they are commonly isolated from the bone marrow or adipose tissue. Bone marrow-derived MSCs (BM-MSCs) were the first MSCs to be discovered and to date are also the most studied in terms of characterization and clinical applications [4]. However, adipose tissue-derived MSCs (AT-MSCs) offer the operational advantages of easier and safer isolation procedures, collection of larger volume of tissue material and higher proliferative capacity [1,5]. BM-MSCs and AT-MSCs have several differences in their characteristics, functions and clinical applications, despite sharing a few similarities [6,7].

In recent decades, the role of MSCs in cancer has generated interest and several studies have been carried out to that end, but the results have been controversial. Some studies attribute both AT-MSCs and BM-MSCs to promoting tumor progression and metastasis $[6,8,9]$. Cells co-expressing MSC markers CD73, CD90 and CD105 were reported to be enriched in head and neck squamous cell carcinoma [10]. MSC-derived factors act on cancer cells to release cytokines, inflammatory mediators and angiogenic factors, and simultaneously inhibit immune cell function [11]. However, there are also studies that indicate tumor-suppressing activities of MSCs. He and colleagues reported that conditioned media from umbilical cord MSCs inhibited breast cancer cell growth, migration and metastasis [12]. BM-MSCs were reported to inhibit angiogenesis and induce apoptosis [13,14]. Nevertheless, the immunosuppressive function of MSCs in general is not always observed, as it depends on variables such as the cell dose, proximity to immune cells, and stimulation by inflammatory cytokines [15]. Given that MSCs are often branded as a promising therapeutic tool, it is necessary to examine MSCs from different sources and in different contexts to understand their biological functions and evaluate their clinical prospects better.

Using a 3-dimensional (3D) myoma organotypic invasion model, we have previously investigated the interaction of BM-MSCs with oral tongue squamous cell carcinoma (OTSCC) cell lines, focusing on the analysis of invasion and gene expression profiles. Our results demonstrated that BM-MSCs were able to promote the invasion of OTSCC cells via inducing the expression of genes linked to chemokine signaling, epithelial plasticity, cell motility and invasion [16].

In this study, we first analyzed the effect of conditioned media from AT-MSCs and BM-MSCs on global long interspersed element-1 (LINE-1) methylation levels in human tongue squamous carcinoma (HSC-3) cells, and the same for HSC-3-derived conditioned media on AT-MSCs and BM-MSCs (Figure 1). LINE-1 is a repetitive DNA element comprising nearly a fifth of the human genome and its methylation level may reflect global genomic methylation [17]. Global LINE-1 methylation has been observed to precede tumor development and may serve as an early indicator of cancer [18]. In parallel, we analyzed changes in expression of intercellular adhesion molecule 1 (ICAM1), integrin alpha-3 (ITGA3) and matrix metallopeptidase 1 (MMP1) expression in AT-MSCs, BM-MSCs and HSC-3 cells in this bidirectional study. ICAM1, ITGA3 and MMP1 are involved in the remodeling of the extracellular matrix, and their expression is known to be aberrant in some cancers [19-21]. We also examined the tumorigenic potential of AT-MSCs on OTSCC by evaluating the proliferation, migration and invasion of HSC-3 and dysplastic oral keratinocyte (DOK) cells. 
CONDITIONED MEDIA
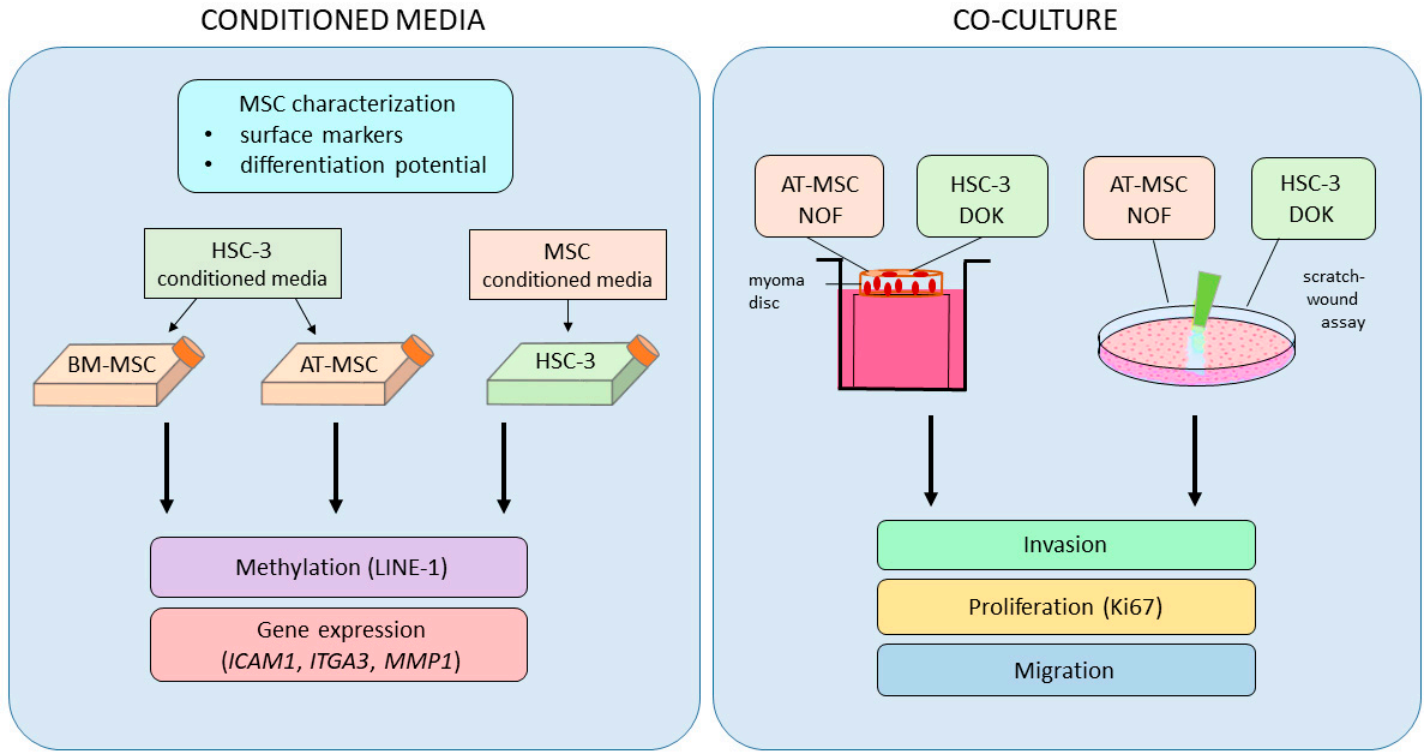

Figure 1. Study design workflow. Adipose tissue-derived mesenchymal stem cells (AT-MSCs) and bone marrow-derived mesenchymal stem cells (BM-MSCs) were first characterized and then used for conditioned media studies with human tongue squamous carcinoma (HSC-3) cells. Cells growing in conditioned media were analyzed for long interspersed nuclear element-1 (LINE-1) methylation and changes in expression of microenvironment remodeling genes. AT-MSCs, HSC-3 cells, dysplastic oral keratinocyte (DOK) cells, and normal oral fibroblasts (NOFs) were used in a co-culture system for invasion, proliferation and migration studies.

\section{Results}

\subsection{HSC-3- and MSCs-Conditioned Media had no Effect on LINE-1 Methylation}

In order to evaluate whether the methylation profile of HSC-3 cells could be affected by contents of the secretome (in conditioned media) from AT-MSCs and BM-MSCs, methylation analysis on a specific site of the LINE-1 element was performed. While the methylation-specific multiplex ligation-dependent probe amplification (MS-MLPA) assay used here includes three methylated sites in LINE-1, the result here was based on the site that is used in most LINE-1 methylation assays. HSC-3 cells were grown in conditioned media from AT-MSCs $(n=2)$ and BM-MSCs $(n=2)$ for four days, following which DNA was extracted for MS-MLPA. Average LINE-1 methylation in HSC-3 cells grown in both MSCs-conditioned media was marginally lower than that in HSC-3 cells grown in serum-free media (baseline, Figure 2A). Though conditioned media were taken from only two donors per MSC type, none of the conditioned media were indicated to have had any effect on LINE-1 methylation in HSC-3 cells. In the reverse setup, where AT-MSCs $(n=4)$ and BM-MSCs $(n=4)$ were grown in HSC-3-conditioned media for four days, the average LINE-1 methylation level in both MSC types grown in HSC-3-conditioned media was comparable to baseline LINE-1 methylation, based on cells grown in serum-free media (Figure 2B).

\subsection{Microenvironment Remodeling genes Expression of HSC-3 and MSCs Cells were Mainly Unaffected when} Cultured in MSCs- or HSC-3-Conditioned Media

We then investigated whether conditioned media from AT-MSCs and BM-MSCs could affect the expression of genes involved in the microenvironment remodeling of HSC-3 cells. HSC-3 cells were grown in conditioned media from AT-MSCs $(n=2)$ and BM-MSCs $(n=2)$ for four days and evaluated for the expression of ICAM1, ITGA3 and MMP1 by quantitative real-time PCR (qRT-PCR) (Figure 3; Supplementary Table S1). ICAM1 and ITGA3 expression in HSC-3 cells growing in both types of MSCs-conditioned media was comparable to that of cells growing in serum-free media (control). 
MMP1 expression was slightly higher in HSC-3 cells when grown in AT-MSC and BM-MSC media (around 1.5- and 2.5-fold, respectively) compared to the control.
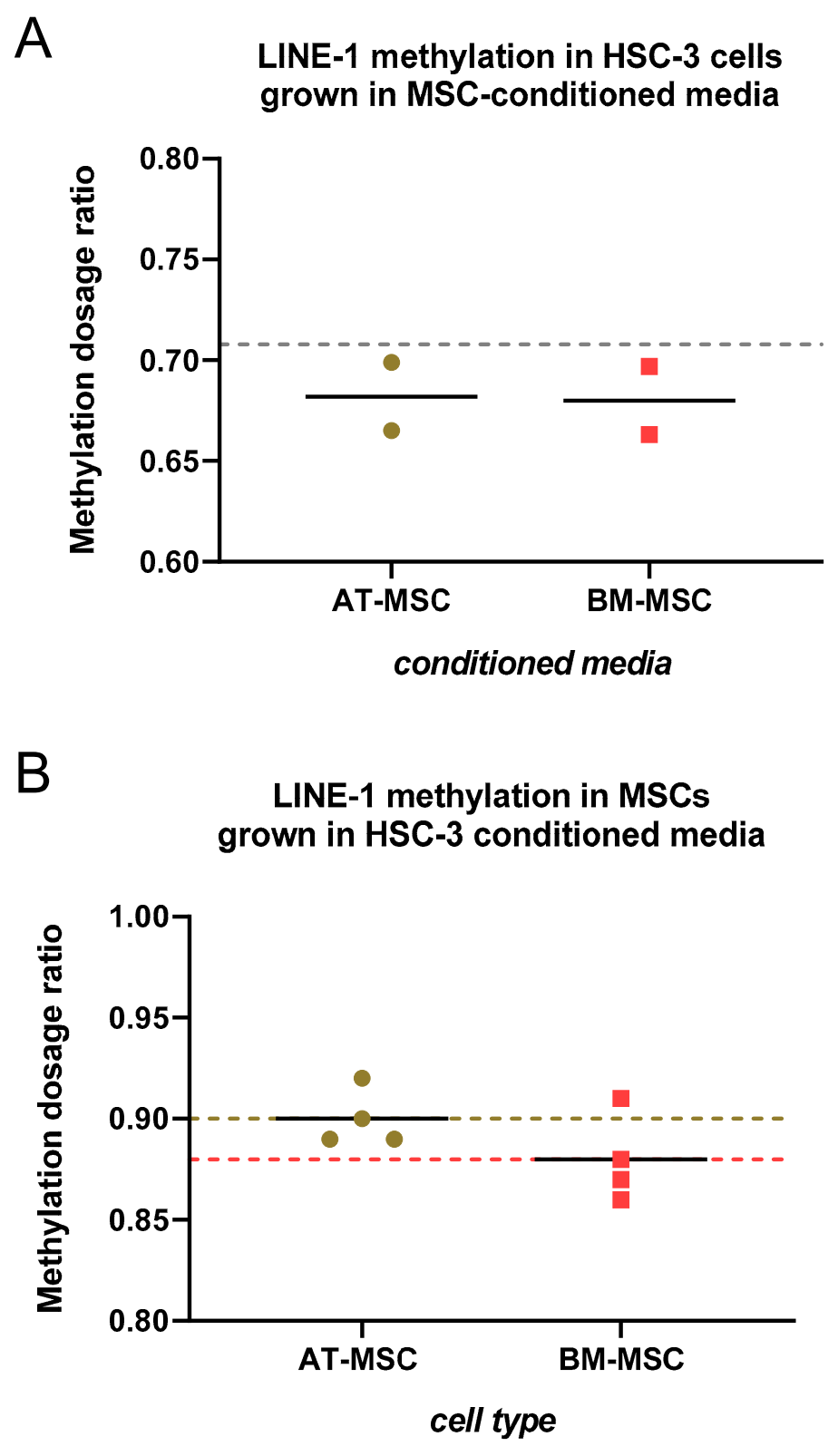

Baseline methylation

-..- AT-MSC

-..- BM-MSC

Figure 2. Effect of HSC-3- and mesenchymal stem cells (MSCs)-conditioned media on LINE-1 methylation in target cells. (A) HSC-3 cells were grown in (AT- and BM-) MSCs-derived conditioned media. DNA was isolated from cells and LINE-1 methylation was analyzed by methylation-specific multiplex ligation-dependent probe amplification (MS-MLPA). The dashed line, baseline methylation, is based on HSC-3 cells grown in serum-free media. (B) AT-MSCs (olive green) and BM-MSCs (red) were grown in HSC-3-derived conditioned media. DNA was isolated from cells and LINE-1 methylation was analyzed by MS-MLPA. Dashed lines represent baseline methylation for each cell type, based on MSCs growing in serum-free media. 

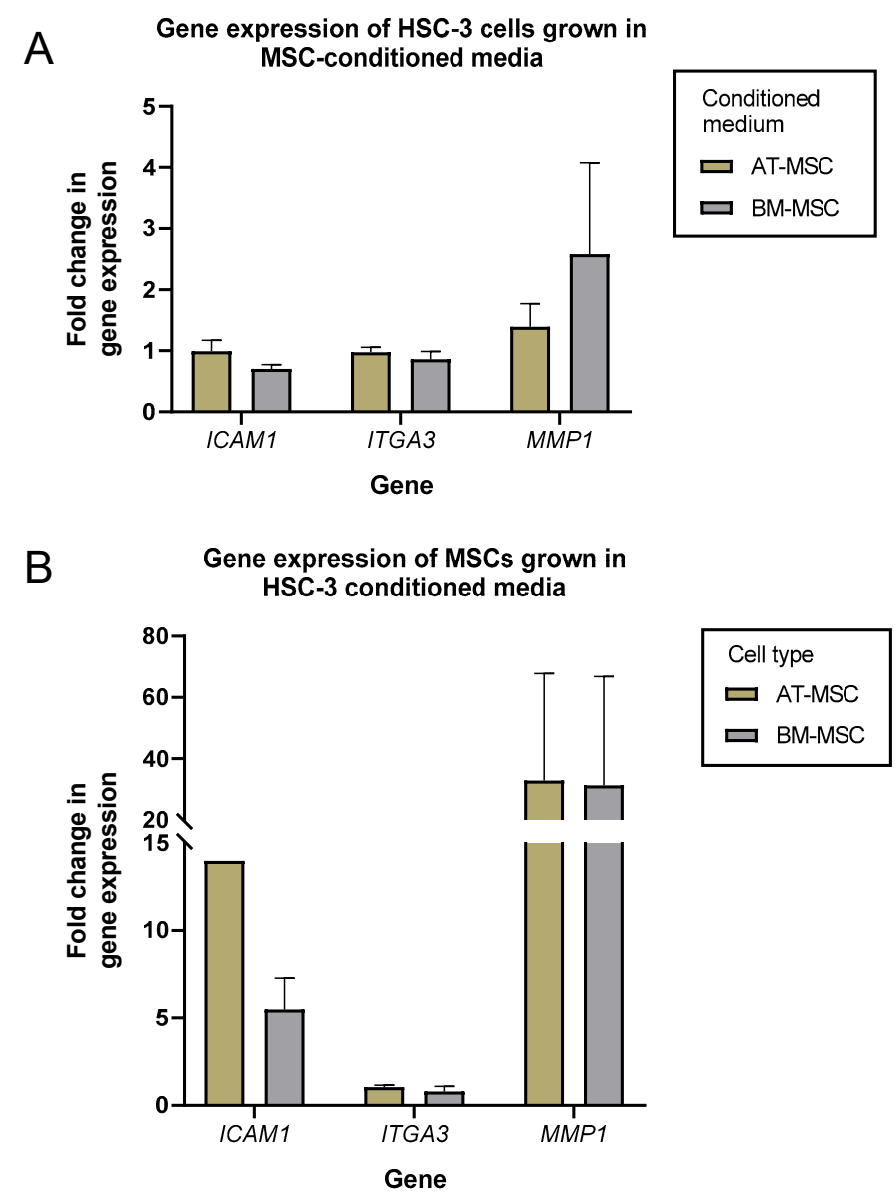

Figure 3. ICAM1, ITGA3 and MMP1 gene expression of HSC-3 and MSCs cells were mainly unaffected when cultured in MSCs- or HSC-3-conditioned media. HSC-3 cells were grown in (AT- and BM-) MSCs-derived conditioned media (A), while AT-MSCs and BM-MSCs were grown in HSC-3-derived conditioned media (B). RNA was isolated from cells and reverse-transcribed to cDNA, then analyzed for changes in gene expression of ICAM1, ITGA3 and MMP1. Fold-change values were normalized to expression levels of the same cells grown in serum-free media.

In a similar way, AT-MSCs $(n=2)$ and BM-MSCs $(n=2)$ were grown in HSC-3-derived conditioned media for four days, and expression levels of the same genes were analyzed (Figure 3B; Supplementary Table S2). Here, only ITGA3 expression was comparable to the control. ICAM1 levels were 14- and 5-fold higher in AT-MSCs and BM-MSCs, respectively, whereas MMP1 levels were higher by 6- to 60-fold in both cell types.

\subsection{AT-MSCs had no Effect on the Invasion and the Proliferation Abilities of HSC-3 or DOK Cells}

Since BM-MSCs were previously shown to induce oral cancer growth, we investigated if AT-MSCs have similar effects. We co-cultured HSC-3 and DOK cells with AT-MSCs and normal oral fibroblasts (NOFs) as the positive control using a 3D myoma disc organotypic model. Both the invasion area and depth were measured using ImageJ software based on pan-cytokeratin staining. Neither the invasion area nor the depth of invasion of HSC-3 and DOK cells were affected by adding AT-MSCs. On the other hand, NOFs, as a control, increased the invasion area and the depth of both HSC-3 and DOK. The increase in the invasion surface area reached statistical significance (Figure 4). Similarly, the number of the small tumor cell islands, budding, was increased when NOFs were added (Figure 4F,G). 

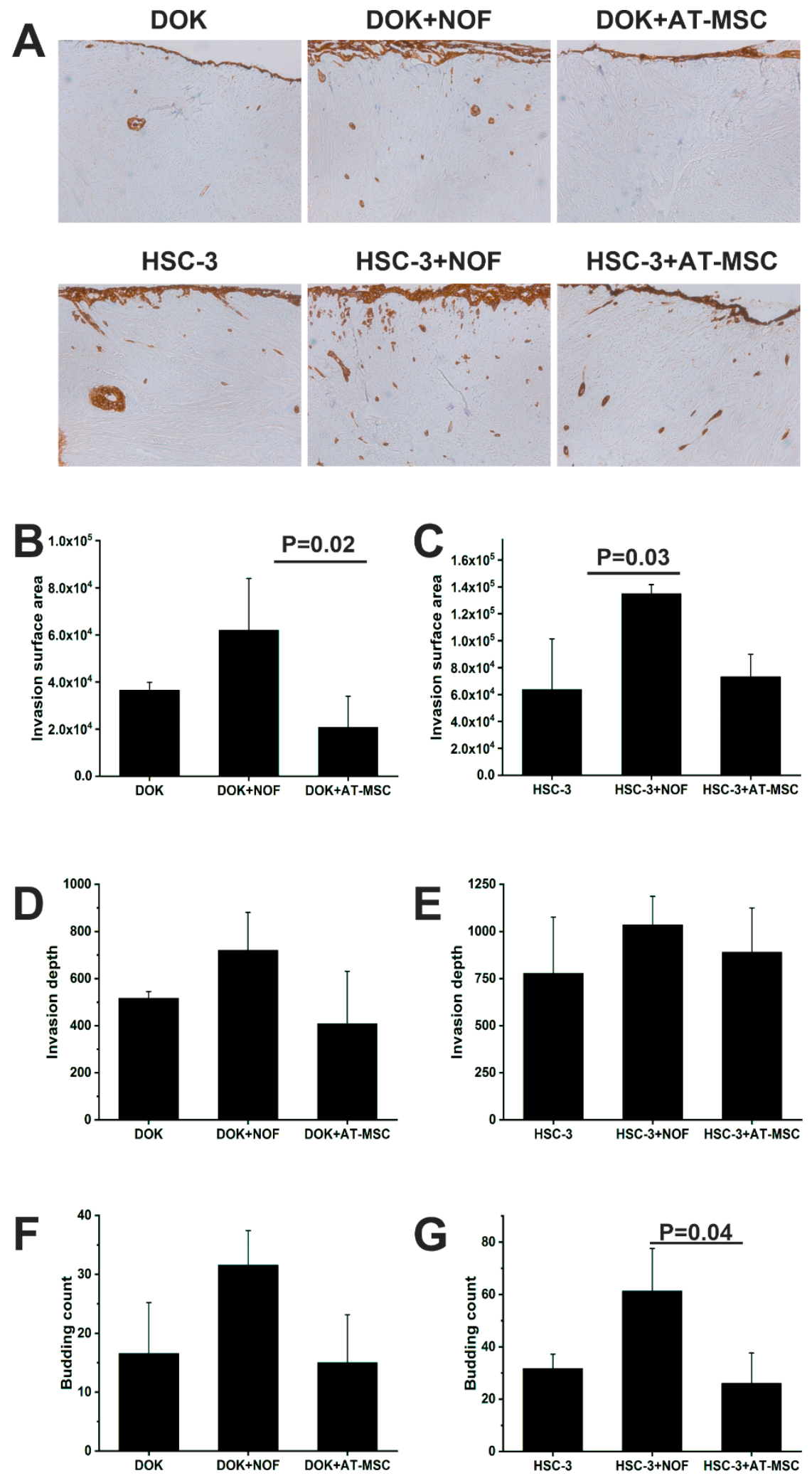

Figure 4. Invasion of DOK and HSC-3 cells was not affected by the presence of AT-MSCs. (A) Representative pictures of the pan-cytokeratin-stained cells in the myoma discs, pictures were taken at magnification $4 \times$. DOK $(\mathbf{B}, \mathbf{D}, \mathbf{F})$ and HSC-3 (C,E,G) cells were co-cultured with AT-MSCs and NOF using myoma organotypic model. Cells' invasion and their ability to form budding were studied. 
To investigate if AT-MSCs have an effect on cancer cells' proliferation, myoma samples were stained with Ki67 and the percentage of the positive cells was calculated. Neither AT-MSCs nor NOFs increased the proliferation ability of the cancer cells (Figure 5).
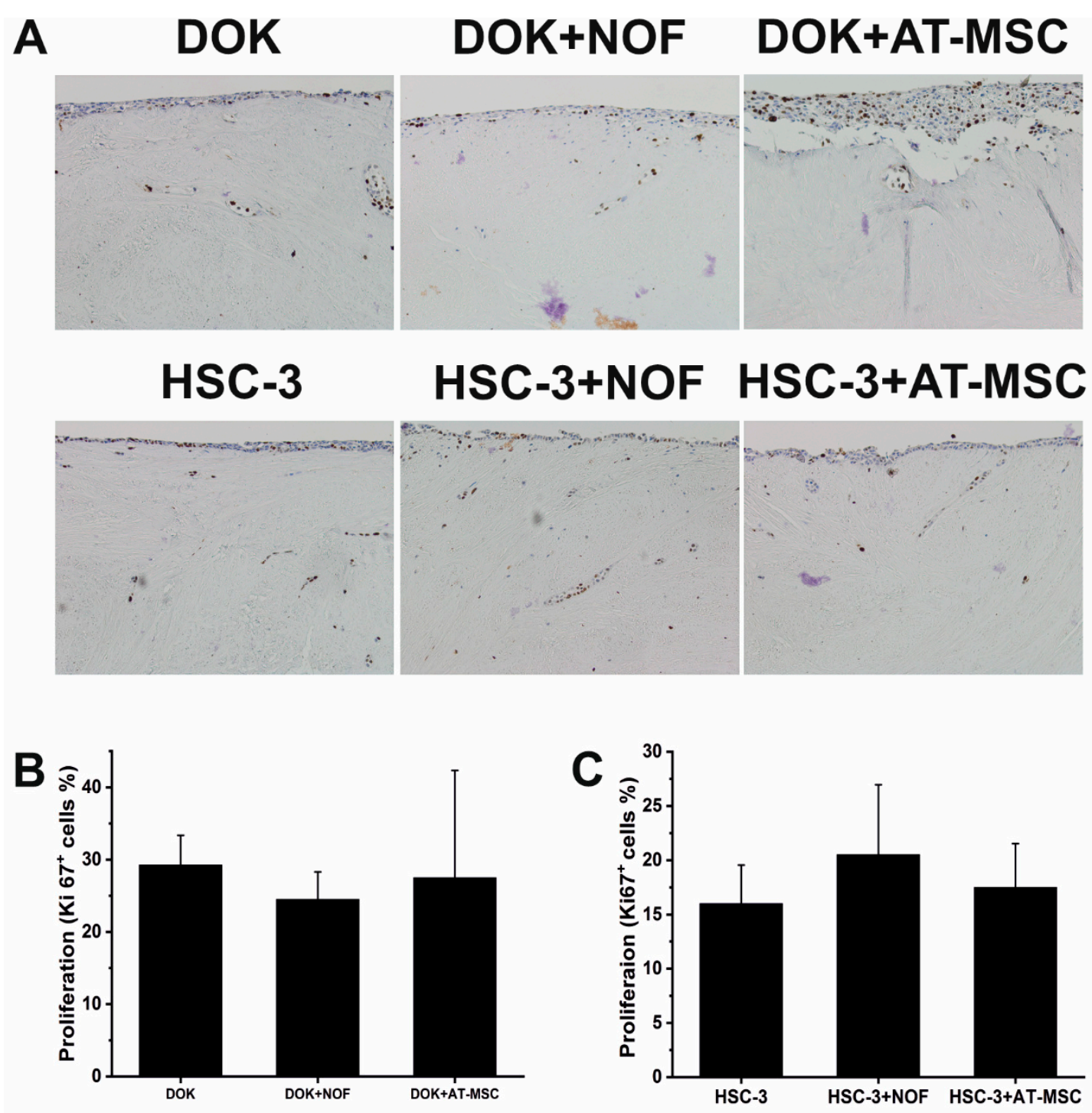

Figure 5. Proliferation of DOK and HSC-3 was not affected by the presence of AT-MSCs. (A) DOK and HSC-3 cells were co-cultured with AT-MSCs and NOF using a myoma organotypic model, pictures were taken at magnification 10×. (B,C) Ki67 was used as a marker for cell proliferation.

\subsection{AT-MSCs and NOF did not Increase the Migration of HSC-3/DOK Cells}

As cancer cells migration is an important feature for cancer progression, we used an IncuCyte wound healing migration assay to study the effect of AT-MSCs and NOFs on the cancer and dysplastic cells migration ability. HSC-3 and DOK were co-cultured with AT-MSCs and NOFs, and a wound was made using a WoundMaker ${ }^{\mathrm{TM}}$ tool. The wound closure rate was monitored for $24 \mathrm{~h}$. Neither AT-MSCs nor NOFs increased, but rather decreased, the migration of HSC-3 and DOK cells (Figure 6). 

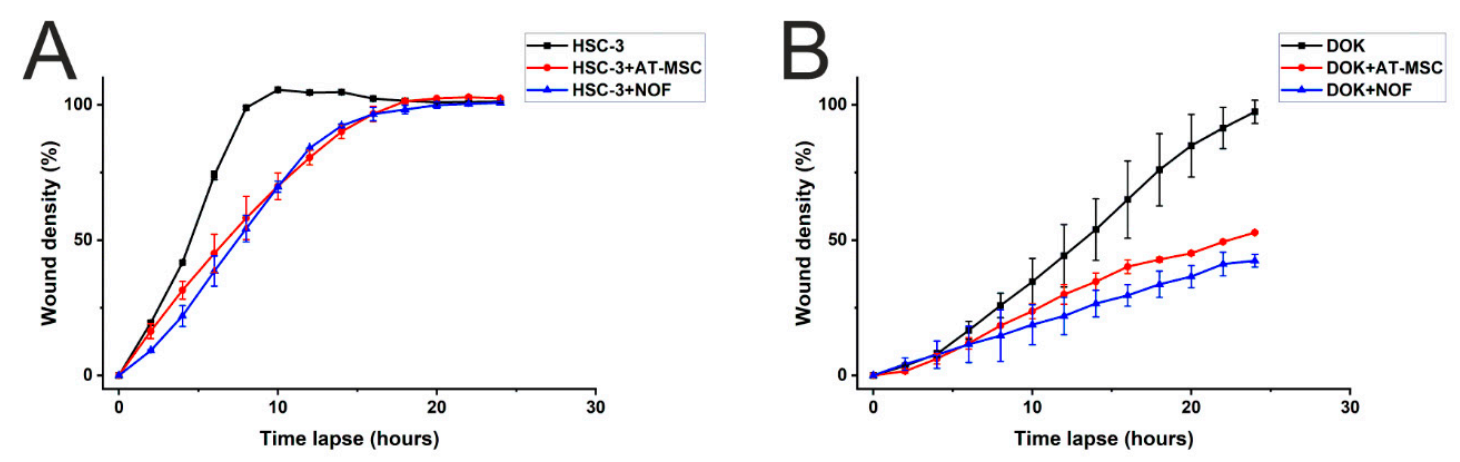

Figure 6. AT-MSC and NOF did not increase the migration of HSC-3 and DOK cells. IncuCyte wound migration assay was conducted to study the effect of AT-MSC and NOF on the migration of (A) HSC-3 cells and (B) DOK cells.

\section{Discussion}

AT-MSCs and BM-MSCs share several biological features but also have a considerable number of differences altogether, therefore they are expected to differ in their functional effects. Initially, differences in their operational features, such as frequency in isolated tissue, ease of harvest, differentiation potential and proliferative capacity, may be considered for their use in a clinical context. Yet, differences in their behavior in tissue and in vivo environments require greater attention. Overall functions of MSCs include regulation of tissue homeostasis and support of tissue integrity. In general, MSCs have an innate tendency to migrate towards damaged tissue and wounds to promote regenerative activities [22,23].

With regard to cancer, it is known that tumor-derived cytokines and other soluble factors attract MSCs towards the tumor microenvironment, thereby playing a vital role in determining the tumor fate [24]. Tumor-associated MSCs have the ability to modulate the tumor microenvironment, since they can exert either stimulatory or inhibitory effects on tumor pathology [6]. For example, conditioned media from AT-MSC reduced the metabolic activity and viability of renal and bladder carcinoma, but also increased the cancer cells' resistance to chemotherapy [25]. Effects of MSCs are also context-dependent: one study reported anti-tumor effects of BM-MSCs on lung cancer and esophageal cancer in vitro, yet they were found to enhance tumor growth in vivo in the same study [26]. With current evidence suggesting that AT-MSCs and BM-MSCs show both anti- and pro-tumor effects in various cancers, this dual function of MSCs could be influenced by several factors, such as: MSCs origin and source, cancer type and the secretome of MSCs and its interaction with the host immune system and other cells in the tumor microenvironment.

Our findings showed that conditioned media from both AT-MSCs and BM-MSCs had very subtle effects on the LINE-1 methylation of HSC-3 cells, and the same when AT-MSCs and BM-MSCs were grown in conditioned media derived from HSC-3. Hypomethylation of LINE-1 is a common phenomenon in many cancers [27], and was also observed in one of our recent studies where AT-MSCs were treated with osteosarcoma-derived extracellular vesicles (EVs) [28]. Those EVs were found to influence AT-MSCs at both the genetic and epigenetic level. In this study, however, no significant changes in methylation level were observed with the conditioned media.

The conditioned media from HSC-3 cells induced upregulation of ICAM1 and MMP1 in both ATand BM- MSCs. ICAM1 expression was upregulated to a higher degree in AT-MSCs than in BM-MSCs, while MMP1 had comparable levels of upregulation in both AT- and BM-MSCs. ICAM1 facilitates intercellular interactions between cells and is implicated in promoting the invasiveness and metastatic ability of cancers $[29,30]$. Meanwhile, MMP1 has been shown to be necessary for the migration of BM-MSCs toward gliomas [31]. MMP1 and other matrix metalloproteinases (MMPs) are upregulated in OTSCC and promote invasion [32,33]. Lastly, no changes in ITGA3 expression were observed in any of the cells, though it has been reported to be over-expressed in many cancers [20]. Though normal 
MSCs did not affect gene expression or LINE-1 methylation in HSC-3 cells, it is possible that MSCs conditioned with HSC-3 media may have pro-tumor effects on HSC-3 cells.

Proliferation, migration, invasion and budding formation are important features needed for cancer progression. AT-MSCs have previously been reported to promote proliferation, invasion and MMPs secretion in epithelial ovarian cancer [34]. Additionally, we have reported that BM-MSCs reduced the proliferation and enhanced the invasion of OTSCC [16]. In this study, however, we were not able to detect similar effects of AT-MSCs on HSC-3 and DOK cells. Conditioned media from AT-MSCs also had minimal effects on MMP1 expression in HSC-3 cells. This clearly shows the differences in the behavior of MSCs based on their origin and the target cells. These results point out that in contrast to BM-MSCs, AT-MSCs do not enhance the aggressiveness of oral dysplastic and cancer cells in vitro.

MSCs offer much promise in regenerative medicine, yet the controversies surrounding the functional effects in vivo and safety concerns hinder their widespread approval. At the moment, AT-MSCs are not as popular as BM-MSCs for therapeutic applications, but their relatively easier harvesting procedures and greater yield from tissues are attractive features that have contributed to a growing interest in AT-MSCs. Nevertheless, functional and clinical studies are of essence to determine the safety and efficiency of AT-MSCs, before they can be established as a viable alternative to BM-MSCs. Furthermore, donor-linked features introduce heterogeneities in MSC behavior, therefore quality control assessments would be necessary to deem them fit-for-purpose. This study indicated that AT-MSCs do not enhance the invasive or migratory potential of OTSCC cells, nor do they influence the expression of microenvironment-remodeling genes in OTSCC. Hence, AT-MSCs are suggested to be more benign than BM-MSCs against OTSCC, with previous findings in consideration. However, this study consisted of a limited number of samples and in vitro investigations, with only OTSCC cells as a target. Analyses of other functional effects and long-term monitoring of in vivo studies are required before making a case for the use of AT-MSCs in clinical applications.

\section{Materials and Methods}

\subsection{Cell Cultures}

Human AT-MSCs were derived from surplus adipose tissue received from plastic surgery procedures at the Department of Plastic Surgery (Laser Tilkka Ltd., Helsinki, Finland). The study was carried out under approval of the ethical committee of Helsinki and Uusimaa Hospital District and with informed consent from the donors (Ethical approval DNro: 217/13/03/02/2015 and HUS/3061/2019). AT-MSCs were obtained from liposuction aspirates from donors using mechanical and enzymatic isolation as described previously [35]. All donors were female, age range 37-54 years, average 46.4 years; body mass index (BMI) range $24-32 \mathrm{~kg} / \mathrm{m}^{2}$, average $26.7 \mathrm{~kg} / \mathrm{m}^{2}$. Cells were cultured in AT-MSCs media consisting of Dulbecco's modified Eagle's medium (DMEM)/Ham's Nutrient Mixture F-12 with 1\% L-alanyl-L-glutamine (DMEM/F-12 1:1 GlutaMAX; Gibco, Paisley, UK), 1\% antibiotics (100 U/mL penicillin, $0.1 \mathrm{mg} / \mathrm{mL}$ streptomycin; Lonza, Verviers, Belgium) and $10 \%$ Fetal Bovine Serum (FBS, South American, Gibco). Cells were grown at $+37^{\circ} \mathrm{C}$ with $5 \% \mathrm{CO}_{2}$ and under humid conditions. In this study, AT-MSCs were in passage 2-6. Once AT-MSCs adhered to the culture flask surface, non-adherent populations were washed away with PBS, and fresh media were added.

Human BM-MSCs were received as a kind gift from Assoc. Prof. Susanna Miettinen (University of Tampere, Tampere, Finland). Donors consisted of three male and one female; age range 69-92, average 84.5; BMI range 19-29, average 24.7. These cells were cultured similarly to AT-MSCs. Cells were in passage $3-4$.

HSC-3 cells (Japan Health Sciences Foundation, Tokyo, Japan) cells and DOK (Sigma-Aldrich, St. Louis, MO, USA) were cultured using DMEM/F-12 (Gibco) supplied with 10\% heat-inactivated FBS, $100 \mathrm{U} / \mathrm{mL}$ penicillin, $100 \mu \mathrm{g} / \mathrm{mL}$ streptomycin, $50 \mu \mathrm{g} / \mathrm{mL}$ ascorbic acid and $250 \mathrm{ng} / \mathrm{mL}$ fungizone (all from Sigma-Aldrich). NOFs were established from tissue explants as described previously [36] and 
cultured using DMEM supplemented with 10\% FBS, $100 \mathrm{U} / \mathrm{mL}$ penicillin, $100 \mu \mathrm{g} / \mathrm{mL}$ streptomycin, $250 \mathrm{ng} / \mathrm{mL}$ fungizone, $1 \%$ sodium pyruvate (Sigma-Aldrich) and $50 \mu \mathrm{g} / \mathrm{mL}$ ascorbic acid.

\subsection{MSCs Characterization}

The MSCs were characterized according to the International Society Cell \& Gene Therapy (ISCT) guidelines, i.e., plastic-adherence, specific surface marker expression and multipotent differentiation potential [37]. The multipotentiality of these cells has been reported in our previous papers [38,39]. AT-MSCs $(n=5)$ and BM-MSCs $(n=4)$ were characterized using a BD Accuri C6 flow cytometer (Becton Dickinson, Franklin Lakes, NJ, USA) to confirm the mesenchymal origin of the cells (Table 1). Allophycocyanin-conjugated monoclonal antibodies against CD14, CD19, CD34, CD45RO, CD54, CD73, CD90, CD105 and HLA-DR (Becton Dickinson) were used to stain at least 100,000 cells per antibody. Analysis was performed for 10,000 events with positive expression defined as the level of fluorescence greater than $99 \%$ of the corresponding unstained cell sample.

Table 1. Characterization of AT-MSCs and BM-MSCs by surface marker expression with flow cytometry analysis. Clone information for marker antibodies is also provided.

\begin{tabular}{cccc}
\hline \multirow{2}{*}{ Marker } & Clone & \multicolumn{2}{c}{$\begin{array}{c}\text { \% Expression in Cells } \\
\text { Average } \pm \text { Standard Deviation) }\end{array}$} \\
\cline { 3 - 4 } & & AT-MSC $(\boldsymbol{n}=\mathbf{5})$ & BM-MSC $(\boldsymbol{n}=\mathbf{4})$ \\
\cline { 3 - 4 } CD14 & M5E2 & $1.7 \pm 2.8$ & $0.4 \pm 0.3$ \\
CD19 & HIB19 & $0.3 \pm 0.4$ & $0.3 \pm 0.2$ \\
CD34 & 581 & $31.9 \pm 25.4$ & $1.1 \pm 1.4$ \\
CD45RO & UCHL1 & $1.4 \pm 2.3$ & $0.5 \pm 0.3$ \\
CD54 & HA58 & $61.8 \pm 22.8$ & $12.4 \pm 2.2$ \\
CD73 & AD2 & $99.0 \pm 1.8$ & $99.5 \pm 0.3$ \\
CD90 & 5E10 & $99.3 \pm 0.7$ & $94.6 \pm 5.1$ \\
CD105 & 266 & $96.1 \pm 7.1$ & $98.2 \pm 1.0$ \\
HLA-DR & G46-6 & $1.7 \pm 3.1$ & $1.5 \pm 0.8$ \\
\hline
\end{tabular}

All nine MSC cell lines were positive for surface markers CD73, CD90 and CD105 in > 90\% of their population, the only exceptions being one AT-MSC cell line with a lower expression of CD105 and one BM-MSC cell line with a slightly lower expression of CD90 (Table 1). MSCs were adherent and displayed multipotent differentiation. AT-MSCs had higher expression of CD34 and CD54 than BM-MSCs. Otherwise, both AT- and BM-MSCs had very low expression of CD14, CD19, CD45 and HLA-DR.

\subsection{Culture of MSCs and HSC-3 Cells in Conditioned Media}

A total of 500,000 cells of each cell type (AT-MSC, BM-MSC and HSC-3) were plated in T-175 flasks (Corning Inc., Corning, NY, USA) and grown until they approached $70 \%$ confluency. Then, the growth media were changed to serum-free conditions (no FBS) and collected after $24 \mathrm{~h}$ of culture. The confluency approach was taken to normalize the metabolite content in the conditioned media. Conditioned media were centrifuged at $600 \times g$, after which the supernatant was passed through a $0.45 \mu \mathrm{m}$ filter and stored at $-80^{\circ} \mathrm{C}$ until use. HSC-3 cells were grown for $24 \mathrm{~h}$, then washed with PBS and grown in (AT- and BM-)MSCs-derived conditioned media for four days. AT-MSC and BM-MSC cells were grown for $24 \mathrm{~h}$ and let to adhere, then washed with PBS and grown in HSC-3-derived conditioned media for four days. In all cases, the same cells were grown in serum-free media as a control.

\subsection{Gene Expression Analysis with $q R T-P C R$}

Total RNA was extracted from AT-MSCs, BM-MSCs and HSC-3 cells using the NucleoSpin ${ }^{\circledR}$ RNA isolation kit (Macherey-Nagel, Düren, Germany) and reverse-transcribed to cDNA using SuperScript ${ }^{\mathrm{TM}}$ 
IV VILO ${ }^{\mathrm{TM}}$ Master Mix (Thermo Fisher Scientific Baltics UAB, Vilnius, Lithuania). All qRT-PCR reactions were conducted in triplicates on MicroAmp ${ }^{\mathrm{TM}}$ Optical 96-well reaction plates using TaqMan assays, with reactions carried out in QuantStudio 5 (all from Life Technologies, Carlsbad, CA, USA). The expression of the following genes was quantified: intercellular adhesion molecule 1 (ICAM1, assay ID Hs00164932_m1), integrin alpha-3 (ITGA3, assay ID Hs01076879_m1) and matrix metallopeptidase 1 (MMP1, assay ID Hs00899658_m1). Expression levels were normalized with the housekeeping gene, ribosomal protein lateral stalk subunit P0 (RPLP0, assay ID Hs99999902_m1), and calculated using the ddCt-method [40].

\subsection{LINE-1 Methylation Analysis}

DNA was extracted from AT-MSCs, BM-MSCs and HSC-3 cells using NucleoSpin ${ }^{\circledR}$ Tissue (Macherey-Nagel). For two samples that had a low cell number, NucleoSpin ${ }^{\circledR}$ Tissue XS (Macherey-Nagel) was used. A total of $90 \mathrm{ng}$ of DNA was used to perform the MS-MLPA reactions, using the SALSA MS-MLPA Reagents kit and P300-B1 Human DNA Reference-2 (MRC-Holland, Amsterdam, The Netherlands). A customized set of LINE-1 probes was used according to a previously established protocol [41,42]. Two controls were used: one commercially available colon cancer cell line RKO (ATCC ${ }^{\circledR}$ CRL-2577 ${ }^{\mathrm{TM}}$, Manassas, VA, USA) and one unmethylated sample generated using the GenomePlex complete whole genome amplification kit. The methylation dosage ratio was calculated as described previously [41].

\subsection{Organotypic Myoma Discs}

In order to study the effect of AT-MSCs on oral cancer (HSC-3) and dysplastic (DOK) cell proliferation and invasion, we have developed an in vitro model using human uterine leiomyoma discs [43]. NOFs were used as the positive control. The use of myoma tissue was approved by the Ethics Committee of Oulu University Hospital, and all patients signed an informed consent form. We prepared uterine myoma discs and completed invasion experiments with some modifications, as described previously $[43,44]$. Briefly, we placed myoma discs into Transwell ${ }^{\circledR}$ inserts (Corning Inc., Corning, NY, USA) and $40 \times 10^{4}$ HSC-3 or DOK with or without $20 \times 10^{4}$ AT-MSCs or NOFs seeded on top of the discs. The cells were allowed to adhere overnight, and on the next day, the discs were transferred onto a steel grid in a 12-well plate supplied with $1 \mathrm{~mL}$ culture media replaced every 3 to 4 days for 10 days. Myoma discs were fixed in 10\% formalin and embedded in paraffin blocks.

\subsection{Immunohistochemical Staining of the Myoma Discs}

Samples were cut into 4- $\mu \mathrm{m}$-thick sections and stained using a fully automated Leica BOND-MAX staining robot (Leica Microsystems, Wetzlar, Germany) and a horseradish peroxidase-labeled dextran polymer method. As for primary antibodies, we used monoclonal mouse anti-human pan-cytokeratin $(0.7 \mu \mathrm{g} / \mathrm{mL})$ and Ki67 (0.8 $\mu \mathrm{g} / \mathrm{mL})$, both from Dako (Dako, Glostrup, Denmark).

\subsection{Scratch-Wound Cell Migration Assay}

To test the effect of AT-MSCs and NOFs on HSC-3 and DOK cell migration, wells of a 96-well Imagelock plate (Essen Bioscience, Ann Arbor, MI, USA) were coated with $300 \mu \mathrm{g} / \mathrm{mL}$ human Myogel matrix [45] and incubated overnight $\left(37^{\circ} \mathrm{C}\right)$. Leftover Myogel was removed by suction and cell suspensions $(100 \mu \mathrm{L} /$ well $)$ were seeded and left to adhere overnight in an incubator. Cell densities were optimized to achieve a confluent cell layer after 24 h; HSC-3 (25,000 cells/well), NOFs (12,500 cells/well), AT-MSCs (12,500 cells/well) and DOK (20,000 cells/well). Cell lines were seeded alone and as a mixture of two cell lines. For preparation of homogeneous wounds, we used a WoundMaker ${ }^{\mathrm{TM}}$ tool (Essen Bioscience). After wound making, cell culture media were replaced with $100 \mu \mathrm{L}$ of fresh media. Wound confluence was monitored using the IncuCyte Zoom Live-Cell Imaging System (Essen Bioscience) by taking images every second hour for $24 \mathrm{~h}$. 


\subsection{Microscopic and Histomorphometric Analysis}

Slides were imaged using a Leica DM6000 B/M light microscope connected to a digital camera (DFC420; Leica Microsystems). We used ImageJ 1.51 (64-bit) software to measure the invasion area, the invasion depth and the average cell island size, budding, as described previously $[43,44,46]$. The percentage of $\mathrm{Ki} 67^{+}$cells was calculated from at least three randomly selected fields of the non-invading cells (on the myoma surface).

\subsection{Statistical Analysis}

When characterizing MSCs from different donors in terms of surface marker expression, values were reported as means \pm standard deviations. Gene expression values were also provided as means \pm standard deviations. Myoma invasion experiments were repeated four times independently, each in duplicate. Values are given as means \pm standard deviations. To determine the statistical significance, we performed one-way analysis of variance (ANOVA) followed by the Bonferroni post hoc test. We set statistical significance to $P<0.05$.

Supplementary Materials: Supplementary materials can be found at http://www.mdpi.com/1422-0067/21/18/ 6455/s1.

Author Contributions: Conceptualization, S.K., B.M., R.S.-K. and A.A.-S.; methodology, S.S., M.N.-S. and K.T.; validation, S.S., M.N.-S. and K.T.; formal analysis, S.S., M.N.-S., K.T. and A.A.-S.; investigation, S.S. and M.N.-S.; resources, T.S. and R.S.-K.; data curation, S.S., M.N.-S., B.M., S.K., T.S. and A.A.-S.; writing original draft preparation, S.S., B.M. and S.K.; writing review and editing, all co-authors; visualization, S.S., M.N.-S. and K.T.; supervision, S.K., B.M., R.S.-K., T.S. and A.A.-S.; project administration, T.S. and R.S.-K.; funding acquisition, T.S. and R.S.-K. All authors have read and agreed to the published version of the manuscript.

Funding: This research was funded by University of Helsinki project funding (WBS490302), the Doctoral Programme in Oral Sciences (FINDOS), the Doctoral Programme in Clinical Research (KLTO), Helsinki University Hospital State funding for university-level health research, Lasten Syöpäsäätiö Väre, the Finnish Dental Society Apollonia, Finnish-Norwegian medical foundation, the Selma and Maja-Lisa Selander Foundation, Sigrid Jusélius Foundation, the Cancer Society of Finland, the Oulu University Hospital MRC grant, and the Jane and Aatos Erkkos Foundation. Open access funding provided by University of Helsinki.

Acknowledgments: Roman Kornilov and Ahmed Abu-Shahba are thanked for flow cytometry analyses, as is the Biomedicum Flow Cytometry Unit. The authors would like to thank Walter Pavicic for providing the LINE-1 probes for methylation analysis. The Sequencing unit of Institute of Molecular Medicine Finland FIMM Technology Centre, University of Helsinki is acknowledged for MS-MLPA fragment analysis service. The FIMM High Throughput Biomedicine Unit is acknowledged for providing the Incucyte ${ }^{\circledR}$ WoundMaker ${ }^{\mathrm{TM}}$ tool.

Conflicts of Interest: The authors declare no conflict of interest. The funders had no role in the design of the study; in the collection, analyses, or interpretation of data; in the writing of the manuscript, or in the decision to publish the results.

\section{Abbreviations}

$\begin{array}{ll}\text { ANOVA } & \text { Analysis of variance } \\ \text { AT-MSC } & \text { Adipose tissue-derived mesenchymal stem cell } \\ \text { BM-MSC } & \text { Bone marrow-derived mesenchymal stem cell } \\ \text { DOK } & \text { Dysplastic oral keratinocyte } \\ \text { EV } & \text { Extracellular vesicle } \\ \text { HSC-3 } & \text { Human tongue squamous carcinoma } \\ \text { ICAM1 } & \text { Intercellular adhesion molecule-1 } \\ \text { ITGA3 } & \text { Integrin alpha-3 } \\ \text { LINE-1 } & \text { Long interspersed nuclear element-1 } \\ \text { MMP } & \text { Matrix metallopeptidase } \\ \text { MSC } & \text { Mesenchymal stem cell } \\ \text { MS-MLPA } & \text { Multiplex ligation-dependent probe amplification } \\ \text { NOF } & \text { Normal oral fibroblast } \\ \text { OTSCC } & \text { Oral tongue squamous cell carcinoma }\end{array}$




\section{References}

1. Strioga, M.; Viswanathan, S.; Darinskas, A.; Slaby, O.; Michalek, J. Same or not the same? Comparison of adipose tissue-derived versus bone marrow-derived mesenchymal stem and stromal cells. Stem Cells Dev. 2012, 21, 2724-2752. [CrossRef]

2. Ridge, S.M.; Sullivan, F.J.; Glynn, S.A. Mesenchymal stem cells: Key players in cancer progression. Mol. Cancer 2017, 16, 31. [CrossRef]

3. Wang, M.; Yuan, Q.; Xie, L. Mesenchymal stem cell-based immunomodulation: Properties and clinical application. Stem Cells Int. 2018, 2018, 3057624. [CrossRef]

4. Gomez-Salazar, M.; Gonzalez-Galofre, Z.N.; Casamitjana, J.; Crisan, M.; James, A.W.; Péault, B. Five decades later, are mesenchymal stem cells still relevant? Front. Bioeng. Biotechnol. 2020, 8, 148. [CrossRef]

5. Kern, S.; Eichler, H.; Stoeve, J.; Klüter, H.; Bieback, K. Comparative analysis of mesenchymal stem cells from bone marrow, umbilical cord blood, or adipose tissue. Stem Cells 2006, 24, 1294-1301. [CrossRef]

6. Zhou, X.; Li, T.; Chen, Y.; Zhang, N.; Wang, P.; Liang, Y.; Long, M.; Liu, H.; Mao, J.; Liu, Q.; et al. Mesenchymal stem cell-derived extracellular vesicles promote the in vitro proliferation and migration of breast cancer cells through the activation of the ERK pathway. Int. J. Oncol. 2019, 54, 1843-1852. [CrossRef] [PubMed]

7. Dmitrieva, R.I.; Minullina, I.R.; Bilibina, A.A.; Tarasova, O.V.; Anisimov, S.V.; Zaritskey, A.Y. Bone marrowand subcutaneous adipose tissue-derived mesenchymal stem cells: Differences and similarities. Cell Cycle 2012, 11, 377-383. [CrossRef]

8. Nishikawa, G.; Kawada, K.; Nakagawa, J.; Toda, K.; Ogawa, R.; Inamoto, S.; Mizuno, R.; Itatani, Y.; Sakai, Y. Bone marrow-derived mesenchymal stem cells promote colorectal cancer progression via CCR5. Cell Death Dis. 2019, 10, 264. [CrossRef] [PubMed]

9. Chu, Y.; You, M.; Zhang, J.; Gao, G.; Han, R.; Luo, W.; Liu, T.; Zuo, J.; Wang, F. Adipose-derived mesenchymal stem cells enhance ovarian cancer growth and metastasis by increasing thymosin beta 4X-linked expression. Stem Cells Int. 2019, 2019, 9037197. [CrossRef] [PubMed]

10. Liotta, F.; Querci, V.; Mannelli, G.; Santarlasci, V.; Maggi, L.; Capone, M.; Rossi, M.C.; Mazzoni, A.; Cosmi, L.; Romagnani, S.; et al. Mesenchymal stem cells are enriched in head neck squamous cell carcinoma, correlates with tumour size and inhibit T-cell proliferation. Br. J. Med. 2015, 112, 745-754. [CrossRef]

11. Lee, H.Y.; Hong, I.S. Double-edged sword of mesenchymal stem cells: Cancer-promoting versus therapeutic potential. Cancer Sci. 2017, 108, 1939-1946. [CrossRef]

12. He, N.; Kong, Y.; Lei, X.; Liu, Y.; Wang, J.; Xu, C.; Wang, Y.; Du, L.; Ji, K.; Wang, Q.; et al. MSCs inhibit tumor progression and enhance radiosensitivity of breast cancer cells by down-regulating STAT3 signaling pathway. Cell Death Dis. 2018, 9, 1026. [CrossRef]

13. Otsu, K.; Das, S.; Houser, S.D.; Quadri, S.K.; Bhattacharya, S.; Bhattacharya, J. Concentration-dependent inhibition of angiogenesis by mesenchymal stem cells. Blood 2009, 113, 4197-4205. [CrossRef]

14. Rhee, K.J.; Lee, J.I.; Eom, Y.W. Mesenchymal stem cell-mediated effects of tumor support or suppression. Int. J. Mol. Sci. 2015, 16, 30015-30033. [CrossRef]

15. Ankrum, J.A.; Ong, J.F.; Karp, J.M. Mesenchymal stem cells: Immune evasive, not immune privileged. Nat. Biotech. 2014, 32, 252-260. [CrossRef]

16. Salo, S.; Bitu, C.; Merkku, K.; Nyberg, P.; Bello, I.O.; Vuoristo, J.; Sutinen, M.; Vähänikkilä, H.; Costea, D.E.; Kauppila, J.; et al. Human bone marrow mesenchymal stem cells induce collagen production and tongue cancer invasion. PLoS ONE 2013, 8, e77692. [CrossRef]

17. Rodriguez, J.; Frigola, J.; Vendrell, E.; Risques, R.-A.; Fraga, M.F.; Morales, C.; Moreno, V.; Esteller, M.; Capellà, G.; Ribas, M.; et al. Chromosomal instability correlates with genome-wide DNA demethylation in human primary colorectal cancers. Cancer Res. 2006, 66, 8462-9468. [CrossRef]

18. Feinberg, A.; Ohlsson, R.; Henikoff, S. The epigenetic progenitor origin of human cancer. Nat. Rev. Genet. 2006, 7, 21-33. [CrossRef]

19. Figenschau, S.L.; Knutsen, E.; Urbarova, I.; Fenton, C.; Elston, B.; Perander, M.; Mortensen, E.S.; Fenton, K.A. ICAM1 expression is induced by proinflammatory cytokines and associated with TLS formation in aggressive breast cancer subtypes. Sci. Rep. 2018, 8, 11720. [CrossRef]

20. Jiao, Y.; Li, Y.; Liu, S.; Chen, Q.; Liu, Y. ITGA3 serves as a diagnostic and prognostic biomarker for pancreatic cancer. Onco. Targets Ther. 2019, 12, 4141-4152. [CrossRef] 
21. Gobin, E.; Bagwell, K.; Wagner, J.; Mysona, D.; Sandirasegarane, S.; Smith, N.; Bai, S.; Sharma, A.; Schleifer, R.; She, J.-X. A pan-cancer perspective of matrix metalloproteases (MMP) gene expression profile and their diagnostic/prognostic potential. BMC Cancer 2019, 19, 581. [CrossRef]

22. Caplan, A.I.; Dennis, J.E. Mesenchymal stem cells as trophic mediators. J. Cell. Biochem. 2006, 98, $1076-1084$. [CrossRef]

23. Rustad, K.C.; Gurtner, G.C. Mesenchymal stem cells home to sites of injury and inflammation. Adv. Wound Care (New Rochelle) 2012, 1, 147-152. [CrossRef]

24. Kansy, B.A.; Dißmann, P.A.; Hemeda, H.; Bruderek, K.; Westerkamp, A.M.; Jagalski, V.; Schuler, P.; Kansy, K.; Lang, S.; Dumitru, C.A.; et al. The bidirectional tumor-mesenchymal stromal cell interaction promotes the progression of head and neck cancer. Stem Cell Res. Ther. 2014, 5, 95. [CrossRef]

25. Maj, M.; Bajek, A.; Nalejska, E.; Porowinska, D.; Kloskowski, T.; Gackowska, L.; Drewa, T. Influence of mesenchymal stem cells conditioned media on proliferation of urinary tract cancer cell lines and their sensitivity to ciprofloxacin. J. Cell. Biochem. 2017, 118, 1361-1368. [CrossRef]

26. Li, L.; Tian, H.; Yue, W.; Zhu, F.; Li, S.; Li, W. Human mesenchymal stem cells play a dual role on tumor cell growth in vitro and in vivo. J. Cell. Physiol. 2011, 226, 1860-1867. [CrossRef]

27. Howard, G.; Eiges, R.; Gaudet, F.; Jaenisch, R.; Eden, A. Activation and transposition of endogenous retroviral elements in hypomethylation induced tumors in mice. Oncogene 2008, 27, 404-408. [CrossRef]

28. Mannerström, B.; Kornilov, R.; Abu-Shahba, A.G.; Chowdhury, I.M.; Sinha, S.; Seppänen-Kaijansinkko, R.; Kaur, S. Epigenetic alterations in mesenchymal stem cells by osteosarcoma-derived extracellular vesicles. Epigenetics 2019, 14, 352-364. [CrossRef]

29. Di, D.; Chen, L.; Wang, L.; Sun, P.; Liu, Y.; Xu, Z.; Ju, J. Downregulation of human intercellular adhesion molecule-1 attenuates the metastatic ability in human breast cancer cell lines. Oncol. Rep. 2016, 35, 1541-1548. [CrossRef]

30. Hou, C.H.; Lin, F.L.; Tong, K.B.; Hou, S.M.; Liu, J.F. Transforming growth factor alpha promotes osteosarcoma metastasis by ICAM-1 and PI3K/Akt signaling pathway. Biochem. Pharmacol. 2014, 89, 453-463. [CrossRef]

31. Ho, I.A.; Chan, K.Y.; Ng, W.H.; Guo, C.M.; Hui, K.M.; Cheang, P.; Lam, P.Y. Matrix metalloproteinase 1 is necessary for the migration of human bone marrow-derived mesenchymal stem cells toward human glioma. Stem Cells 2009, 27, 1366-1375. [CrossRef]

32. Zhong, P.; Liu, L.; Shen, A.; Chen, Z.; Hu, X.; Cai, Y.; Lin, J.; Wang, B.; Li, J.; Chen, Y.; et al. Five extracellular matrix-associated genes upregulated in oral tongue squamous cell carcinoma: An integrated bioinformatics analysis. Oncol. Lett. 2019, 18, 5959-5967. [CrossRef]

33. Liu, X.; Yu, J.; Jiang, L.; Wang, A.; Shi, F.; Ye, H.; Zhou, X. MicroRNA-222 regulates cell invasion by targeting matrix metalloproteinase 1 (MMP1) and manganese superoxide dismutase 2 (SOD2) in tongue squamous cell carcinoma cell lines. Cancer Genom. Proteom. 2009, 6, 131-139.

34. Chu, Y.; Tang, H.; Guo, Y.; Guo, J.; Huang, B.; Fang, F.; Cai, J.; Wang, Z. Adipose-derived mesenchymal stem cells promote cell proliferation and invasion of epithelial ovarian cancer. Exp. Cell Res. 2015, 337, 16-27. [CrossRef]

35. Peltoniemi, H.H.; Salmi, A.; Miettinen, S.; Mannerström, B.; Saariniemi, K.; Mikkonen, R.; Kuokkanen, H.; Herold, C. Stem cell enrichment does not warrant a higher graft survival in lipofilling of the breast: A prospective comparative study. J. Plast. Reconstr. Aesthet. Surg. 2013, 66, 1494-1503. [CrossRef]

36. Sobral, L.M.; Zecchin, K.G.; Nascimento de Aquino, S.; Lopes, M.A.; Graner, E.; Coletta, R.D. Isolation and characterization of myofibroblast cell lines from oral squamous cell carcinoma. Oncol. Rep. 2011, 25, 1013-1020. [CrossRef]

37. Dominici, M.; Le Blanc, K.; Mueller, I.; Slaper-Cortenbach, I.; Marini, F.; Krause, D.; Deans, R.; Keating, A.; Prockop, D.J.; Horwitz, E.M. Minimal criteria for defining multipotent mesenchymal stromal cells. The International Society of Cellular Therapy position statement. Cytotherapy 2006, 8, 315-317. [CrossRef]

38. Lindroos, B.; Boucher, S.; Chase, L.; Kuokkanen, H.; Huhtala, H.; Haataja, R.; Vemuri, M.; Suuronen, R.; Miettinen, S. Serum-free, xeno-free culture media maintain the proliferation rate and multipotentiality of adipose stem cells in vitro. Cytotherapy 2009, 11, 958-972. [CrossRef]

39. Kaur, S.; Abu-Shahba, A.G.; Paananen, R.O.; Hongisto, H.; Hiidenmaa, H.; Skottman, H.; Seppänen-Kaijansinkko, R.; Mannerström, B. Small non-coding RNA landscape of extracellular vesicles from human stem cells. Sci. Rep. 2018, 8, 15503. [CrossRef] 
40. Livak, K.J.; Schmittgen, T.D. Analysis of relative gene expression data using real-time quantitative PCR and the 2(-delta C(t)) method. Methods 2001, 25, 402-408. [CrossRef]

41. Pavicic, W.; Joensuu, E.I.; Nieminen, T.; Peltomäki, P. LINE-1 hypomethylation in familial and sporadic cancer. J. Mol. Med. 2012, 90, 827-835. [CrossRef]

42. Sinha, S.; Mannerström, B.; Seppänen-Kaijansinkko, R.; Kaur, S. LINE-1 methylation analysis in msesnchymal stem cells treated with osteosarcoma-derived extracellular vesicles. J. Vis. Exp. 2020, 156, e60705. [CrossRef]

43. Nurmenniemi, S.; Sinikumpu, T.; Alahuhta, I.; Salo, S.; Sutinen, M.; Santala, M.; Risteli, J.; Nyberg, P.; Salo, T. A novel organotypic model mimics the tumor microenvironment. Am. J. Pathol. 2009, 175, 1281-1291. [CrossRef]

44. Åstrom, P.; Heljasvaara, R.; Nyberg, P.; Al-Samadi, A.; Salo, T. Human tumor tissue-based 3D in vitro invasion assays. Methods Mol. Biol. 2018, 1731, 213-221. [CrossRef]

45. Salo, T.; Sutinen, M.; Hoque Apu, E.; Sundquist, E.; Cervigne, N.K.; De Oliveira, C.E.; Akram, S.U.; Ohlmeier, S.; Suomi, F.; Eklund, L.; et al. A novel human leiomyoma tissue derived matrix for cell culture studies. BMC Cancer 2015, 15, 981. [CrossRef]

46. Dourado, M.R.; Korvala, J.; Åström, P.; De Oliveira, C.E.; Cervigne, N.K.; Mofatto, L.S.; Campanella Bastos, D.; Pereira Messetti, A.C.; Graner, E.; Paes Leme, A.F.; et al. Extracellular vesicles derived from cancer-associated fibroblasts induce the migration and invasion of oral squamous cell carcinoma. J. Extracell Vesicles 2019, 8, 1578525. [CrossRef]

(C) 2020 by the authors. Licensee MDPI, Basel, Switzerland. This article is an open access article distributed under the terms and conditions of the Creative Commons Attribution (CC BY) license (http://creativecommons.org/licenses/by/4.0/). 